largest vertical diameter of the right orbit is $4.6 \mathrm{~cm}$., that of the left $4.1 \mathrm{~cm}$.; the largest horizontal diameters are $4.1 \mathrm{~cm}$. and $3.9 \mathrm{~cm}$. respectively.

We wish to express our indebtedness to the surgical staffs of the Royal Eye Hospital and of Guy's Hospital for permission to follow up cases under their care.

\title{
A DEVICE FOR PREVENTING GLARE IN ELECTRIC OPHTHALMOSCOPES
}

BY

\author{
N. BISHOP HARMAN
}

\begin{abstract}
LONDON
THE introduction of the electric ophthalmoscope has greatly increased facilities for the examination of the interior of the eye, but the internal position of the source of the illumination within the instrument has produced certain new difficulties which the older reflecting ophthalmoscope did not possess. The electric lamp within the stem of the electric ophthalmoscope throws a beam of light parallel to the instrument, and this beam must be bent at right angles to enter the eye of the patient. Prisms and mirrors of various designs have been used to effect this bending. The desideratum is such a complete bending of the rays of light into the eye of the patient that the surgeon has a clear peep-hole through the instrument along the beam of light, and free from all disturbances such as will be caused by rays of light that may be diverted backward from the instrument so as to reach his own eye. If there are such aberrant rays the surgeon cannot see the fundus clearly by reason of the haze that is caused by the rays. The effect is somewhat similar to that disturbance to visibility produced by the rays of the head lamps of a car when there is a slight mist at night. These disturbing effects have become more evident with the increase in power of the light available.

The aberrant rays are brought about by several conditions. If a glass mirror with a drilled hole be used in the ophthalmoscope the beam of light will impinge upon the upper edge of this hole, and no matter how it may be blackened, this edge will scatter a blaze of disturbing light. If the mirror has no drilled hole but only a gap in the silvering, the hinder surface of the glass itself will reflect any light that may fall upon it from above the ophthalmoscope, and the slightest imperfection in the glass or dust upon it will increase the disturbance.
\end{abstract}


To overcome this defect, Marple cut out the upper edge of the mirror and used one of $U$ shape. It was very successful, but the mirror was too fragile. A polished stainless steel mirror of $U$ shape was substituted, but this has two drawbacks in that it has a lower reflecting power, and soon scratches, still further reducing the amount of light reflected. Prisms, such as were used in the first ophthalmoscopes, avoided some of the difficulties, but if they are well designed they are costly and permit very little variation of the beam, while the inexpensive ones are inefficient.

Recently, in discussing electric ophthalmoscopes with Mr. J. W. Tudor Thomas, of Cardiff, he told me that there had come into his possession an instrument, the origin of which he did not know, which

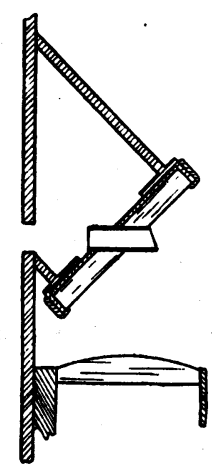

Section through mirror fitted with glare shield.

was free from these defects. A small tube had been fitted into the drilled hole of the glass mirror. This device is the simplest, most natural, and effective correction of the one difficulty of the electric ophthalmoscope. It does exactly what we instinctively do to protect our eyes from the glare. We shield them with our hands, or with hat-brims, or we fit vizors to the wind screens of our cars.

A small thin-walled tube is mounted within the hole of the mirror of the ophthalmoscope, the forward end of the tube is cut at an angle so that there projects from the hole of the mirror a crescentic ledge or shield. This shield fits the lower edge of the drilled hole, and projects just sufficiently to cast a shadow over the hole. When the shield is in position, no aberrant rays can be diverted into the surgeon's eye, and he gets a perfectly clear view into the patient's eye. For a mirror set at an angle of $45^{\circ}$ to the beam from the lamp, and a hole of $2 \mathrm{~mm}$. diameter, the shield fitted to the lower edge of the hole must project $2.5 \mathrm{~mm}$. to provide complete protection. The shield is made of metal, and is fitted to the metal bed-plate of the mirror, but is independent of the mirror glass, so 
that it is secure and not likely to be broken. Also the mirror glass can be detached without disturbing the metal shield.

One small difficulty arose in the use of the device. When the beam from the lamp was exactly centred in the mirror, it was found that the shield cut out the focused rays when they met at the position of the shield. This difficulty was overcome by slightly decentering the lamp, so that the beam when exactly focused on the mirror impinged upon it to one side of the drilled hole. This decentering has no drawback. Messrs. Rayner, of New Bond Street, have fitted this shield to the new electric ophthalmoscope made by them for me, and which was described in your issue of February, 1932. The device is a real advantage, and it will be the standard pattern. It has been fitted to other instruments and has effected a great improvement in each case.

\section{ANNOTATION}

\section{Fishy Vision}

An interesting article, under the above heading, is contributed to the Fishing Gazette of February 20, 1932, by M. S. Mayou, who is a keen fisherman as well as an ophthalmic surgeon, and is thereby doubly qualified to write on this subject. His paper is an admirable exposition which is addressed principally to those fishermen who have no special knowledge of how sight is conducted, but it will not fail to interest the ophthalmic surgeon also. Fish do not have to rely on visual impressions entirely, as cutaneous sensations tell them their position in the stream, the strength of the current and the presence of obstacles such as weed and stones. The cutaneous nerve endings link up with ganglia along the lateral line on either side of the fish, and with a highly developed auditory system.

In investigating the visual powers of fresh-water fish three lines of approach may be used : first, observations on what a fish appears to see; second, the anatomical structure of the fish's eye, optic tracts and brain as compared with those of mammals; and thirdly, the experimentally placing one's own eye under water and estimating from what one sees how much a fish can see. The third method is wholly unreliable, as the eyes and brain of a fish are not constructed as in the human.

Mayou has experimented with perch in a tank. He found that when bits of worm were dropped into the tank without a splash between the fish and the daylight, they were secured by the fish every time; if, on the other hand, the bit was dropped between the 